\title{
Bone Formation With the Combination of Simvastatin and Calcium Sulfate in Critical-Sized Rat Calvarial Defect
}

\author{
Myat Nyan ${ }^{1,2, *}$, Daisuke Sato ${ }^{1}$, Mitsumasa Oda ${ }^{1}$, Tetsu Machida ${ }^{1}$, Hiroshi Kobayashi ${ }^{1}$, Takahiro Nakamura ${ }^{1}$, \\ and Shohei Kasugai ${ }^{1,2}$ \\ ${ }^{I}$ Oral Implantology and Regenerative Dental Medicine, ${ }^{2}$ Center of Excellence Program for Frontier Research on Molecular \\ Destruction and Reconstruction of Tooth and Bone, Tokyo Medical and Dental University, \\ 1-5-45 Yushima, Bunkyo-ku, Tokyo 113-8549, Japan
}

Received April 16, 2007; Accepted June 1, 2007

\begin{abstract}
Simvastatin, a cholesterol synthesis inhibitor, enhances BMP2 expression in osteoblasts. The purpose of the present study was to examine whether simvastatin stimulates bone regeneration when combined with calcium sulfate as a carrier. Critical-sized bone defects in rat calvaria were treated with calcium sulfate or with combination of $1 \mathrm{mg}$ simvastatin and calcium sulfate. In the combination group, although the least amount of bone formation with intense soft tissue inflammation was observed at 2 and 4 weeks, remarkable bone formation was evident at 8 weeks. Conclusively, the combination of simvastatin and calcium sulfate stimulated bone regeneration in spite of the inflammatory response.
\end{abstract}

Keywords: simvastatin, calcium sulfate, bone formation

The demand for use of alloplastic materials is increasing since there are limitations in grafting autogenous bone. To develop more effective bone graft materials with enhanced osteogenic properties, alloplastic materials are combined with osteoinductive substances such as BMPs. Topically applied simvastatin, a cholesterollowering drug, has been shown to stimulate bone growth of mouse calvaria by stimulating BMP-2 $(1,2)$. Other studies demonstrated that local application of statins with different carriers induced osteogenesis and bone growth $(3-6)$. Calcium sulfate is highly biocompatible, bioresorbable, and osteoconductive; and its potential as a carrier for local release of antibiotics and growth factors has been reported $(7-13)$. Thus, the combination with simvastatin and calcium sulfate seems to be attractive as a new bone substitute enhancing bone growth. In the present study, we examined the effects of this combination on healing of critical-sized bone defect in the rat.

This study was approved by the institutional committee for animal experiments. Simvastatin powder (1 mg) (Merck \& Co., Inc., Whitehouse Station, NJ, USA) was incorporated into $60 \mathrm{mg}$ calcium sulfate powder (Grade

*Corresponding author. myatnyan@gmail.com

Published online in J-STAGE

doi: 10.1254/jphs.SC0070184 for thin layer chromatography; Wako Pure Chemical Industries, Ltd., Osaka). The powder was mixed with sterilized distilled water in a ratio of 1:0.4. Then, the mixture was poured in a template to form a disc of $8-\mathrm{mm}$ diameter and 1-mm thickness. Calcium sulfate discs of the same dimension were prepared without simvastatin incorporation. Forty-five male Wistar rats (14-week-old) were used divided into three groups. Under anesthesia with a combination of ketamine $(40 \mathrm{mg} / \mathrm{kg})$ - xylazine $(5 \mathrm{mg} / \mathrm{kg})$, a flap was raised and a bone defect of 8-mm diameter was created with a bone trephine bur. In the three groups, the defect was treated differently: no treatment, calcium sulfate, and the combination of simvastatin and calcium sulfate. The flap was repositioned and sutured. The animals were sacrificed at 2, 4, and 8 weeks after the surgery. The calvaria were dissected out and examined with soft X-ray radiography and dual-energy X-ray absorptiometry for small animals (DCS-600; Aloka Co., Ltd., Tokyo). They were further analyzed with micro CT (InspeXio; Shimadzu Science East Corporation, Tokyo) and the bone volume in the defect area was measured. The differences in morphometric values of the three groups at each time point were analyzed with One Way ANOVA and Scheffe post-hoc multiple comparison tests.

In calcium sulfate-simvastatin group, the skin over 
Table 1. Measurement of bone at the defect sites

\begin{tabular}{|c|c|c|c|c|c|c|c|c|c|}
\hline & \multicolumn{3}{|c|}{2 weeks } & \multicolumn{3}{|c|}{4 weeks } & \multicolumn{3}{|c|}{8 weeks } \\
\hline & Control & C.S & C.S + statin & Control & C.S & C.S + statin & Control & C.S & C.S + statin \\
\hline $\mathrm{BA}\left(\mathrm{cm}^{2}\right)$ & $0.88 \pm 0.2$ & $1.09 \pm 0.04 *$ & $0.95 \pm 0.02$ & $0.93 \pm 0.03$ & $0.93 \pm 0.02$ & $0.91 \pm 0.04$ & $0.97 \pm 0.02$ & $0.98 \pm 0.05$ & $1.15 \pm 0.01^{\#}$ \\
\hline $\mathrm{BMC}(\mathrm{mg})$ & $49.6 \pm 2.41$ & $56.3 \pm 2.18$ & $49.9 \pm 2.37$ & $58.7 \pm 1.7^{*}$ & $55.52 \pm 2.1$ & $50.24 \pm 1.87$ & $62.22 \pm 3.1$ & $68.2 \pm 3.26$ & $79.48 \pm 3.23 * *$ \\
\hline $\operatorname{BMD}\left(\mathrm{mg} / \mathrm{cm}^{2}\right)$ & $55.9 \pm 1.73$ & $51.7 \pm 1.6$ & $52.3 \pm 2.04$ & $62.8 \pm 0.62$ & $56.2 \pm 1.44$ & $55.7 \pm 2.95$ & $63.9 \pm 2.08$ & $69.6 \pm 2.08$ & $69.3 \pm 2.45$ \\
\hline
\end{tabular}

One Way ANOVA with Scheffe's post-hoc multiple comparison: ${ }^{*} P<0.05$, ${ }^{*} P<0.01,{ }^{\sharp} P=0.01$, significantly higher than other groups at the same study period. Bone defects were prepared and treated with calcium sulfate (C.S) or with the combination of calcium sulfate and simvastatin (C.S + statin), whereas in the control group, the defects were not treated. Bone area (BA), bone mineral content (BMC), and bone mineral density (BMD) including the defect site were measured with DXA at 2, 4, and 8 weeks after the surgery. Values are expressed as the mean \pm S.E.M. for five animals in each group.

the bone defect site showed intense inflammation (redness and edema) at about one week post-operation, and scab formation occurred by 2 weeks. The inflammation and scabs continued up to 5 weeks, after which they became subsided and healed normally. Calcium sulfate showed only a faint radiopacity in the radiographs obtained at 2 weeks, and it could not be detected in soft $\mathrm{X}$-ray radiographs at 4 weeks in both the calcium sulfate group and calcium sulfate-simvastatin group. At 8 weeks, radio-opaque areas were detected in the calcium sulfate-simvastatin group. According to the analytical values obtained with dual-energy X-ray absorptiometry (DXA), the calcium sulfate-simvastatin group showed the least amount of bone compared to the other groups at 4 weeks. However, at 8 weeks, bone

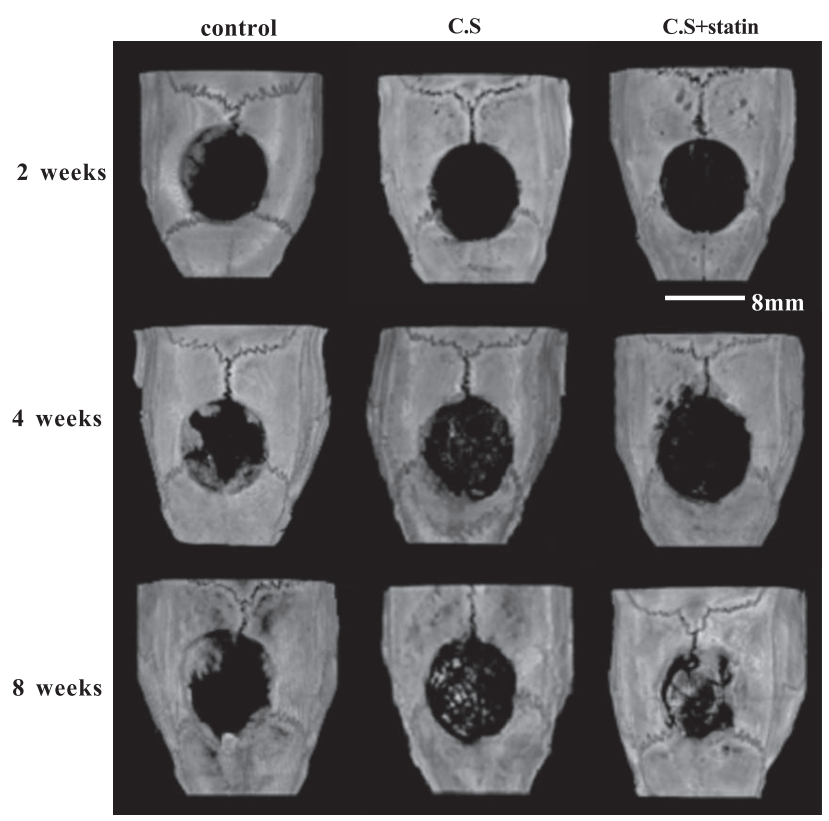

Fig. 1. Micro CT images of the defects sites. Bone defects were prepared and treated with calcium sulfate (C.S) or with the combination of calcium sulfate and simvastatin (C.S + statin); in the control group, the defects were not treated. Micro CT images were taken at 2,4 , and 8 weeks after the surgery. area and bone mineral content of the calcium sulfatesimvastatin group became significantly higher than those of the other groups $(P=0.01$ and $P<0.01$, respectively) (Table 1). Bone volume measured with micro CT also showed the least amount of bone in the calcium sulfate-simvastatin group compared to those of the other groups at 2 and 4 weeks $(P<0.01$, for each). At 8 weeks, bone volume in the calcium sulfate-simvastatin group dramatically increased and it was significantly higher than those of the other groups $(P=0.01$, for each) (Figs. 1 and 2).

Previous studies demonstrated the bone promoting effect of local simvastatin application with different carriers in various animal models. Wong et al. applied collagen sponge containing $0.5 \mathrm{mg}$ simvastatin to parietal bone defects in rabbits and showed tiny but statistically significant stimulation of bone repair (3). We used either bovine atelo-collagen or calcium sulfate to carry $2.0 \mathrm{mg}$ simvastatin and applied them to rat incisor tooth extraction socket. We observed extensive increase in alveolar bone thickness when calcium sulfate

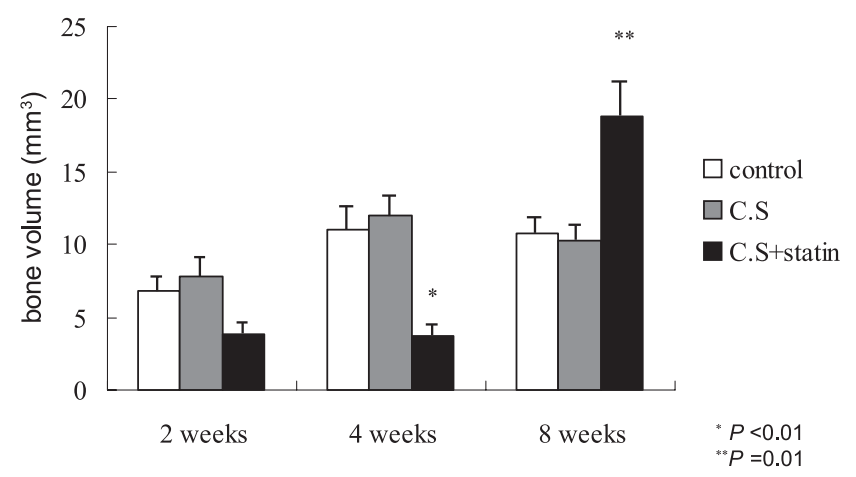

Fig. 2. Bone volume at the defect sites. Bone defects were prepared and treated with calcium sulfate (C.S) or with the combination of calcium sulfate and simvastatin (C.S + statin); in the control group, the defects were not treated. Bone volume at the defect sites were measured with micro $\mathrm{CT}$ at 2, 4, and 8 weeks after the surgery. Values are expressed as the mean \pm S.E.M. for five animals in each group. 
was used as carrier, which suggests the potential of calcium sulfate as simvastatin carrier (4). On the other hand, Thylin et al. applied methylcellulose gel containing $2.2 \mathrm{mg}$ simvastatin with or without polylactide membrane coverage over murine calvaria periosteum. They reported that simvastatin gel with the membrane stimulated bone apposition with inflammation and scabbing of the overlying skin (5). Stein et al. examined the effect of local application of simvastatin $(0.1,0.5$, 1.0 , and $2.2 \mathrm{mg}$ ) with the same methyl cellulose gel and membrane placed over periosteum of rat mandible. In this study each simvastatin dose except $0.1 \mathrm{mg}$ showed significantly more bone area than control group with significant soft tissue inflammation (6). Thus, it seems apparent that local application of simvastatin increases bone, whereas it also elicits inflammation, which is carrier and dose dependent.

In the present study, $1.0 \mathrm{mg}$ simvastatin was combined with calcium sulfate and grafted in rat calvarial defect. Similar to the previous studies, soft tissue inflammation over the defect area was observed from 1 to 5 weeks, albeit without a significant bone formation during this period. The soft tissue inflammation observed exclusively in the calcium sulfate-simvastatin group coincided with the least bone amount in this group among the experimental groups at 2 and 4 weeks. Interestingly, at 8 weeks, when the soft tissue inflammation had completely subsided, a significant amount of bone was observed in the calcium sulfate-simvastatin group.

According to these findings, bone formation in the calcium sulfate-simvastatin group seemed to be delayed as a result of intense soft tissue inflammation, induced by a burst release of simvastatin following an early resorption of calcium sulfate. Bone formation seemed to take place only after 5 weeks when the inflammation in the defect area became subsided. It is not clear whether the previously released simvastatin or that released by a small amount of calcium sulfate, which possibly remained unresorbed after $4-5$ weeks, was responsible for that bone formation. Significant bone formation was not observed in the calcium sulfate group as compared to the control group up to 8 weeks. Calcium sulfate used in this study was the pure grade for thin layer chromatography and it seemed to be resorbed quickly in the bone defect. Much slower resorption of carrier with consequently gradual release of simvastatin is preferable for a faster bone formation in the defect without inducing an intense inflammation. Further studies should be performed using an appropriate carrier scaffold that can release an optimal amount of simvastatin gradually through a slow degradation and also provide an osteoconductive surface over which bone formation can take place.

Conclusively, the $1.0 \mathrm{mg}$ simvastatin and calcium sulfate graft combination appeared to promote bone formation in the critical-sized rat calvarial defect at 8 weeks after a significant soft tissue inflammation over the defect area up to 5 weeks.

\section{References}

1 Mundy G, Garrett R, Harris S, Chan J, Chen D, Rossini G, et al. Stimulation of bone formation in vitro and in rodents by statins. Science. 1999;286:1946-1949.

2 Garrett IR, Gutierrez G, Mundy GR. Statins and bone formation. Curr Pharm Des. 2001;7:715-736.

3 Wong RWK, Rabie ABM. Statin collagen grafts used to repair bone defects in the parietal bone of rabbits. Br J Oral Maxillofacial Surg. 2003;41:244-248.

4 Sato D, Nishimura K, Ishioka T, Kondo H, Kuroda S, Kasugai S. Local application of simvastatin to rat incisor socket: carrierdependent effect on bone augmentation. J Oral Tissue Engineering. 2005;2:81-85.

5 Thylin MR, McConnell JC, Schmid MJ, Reckling RR, Ojha J, Bhattacharyya I, et al. Effects of statin gels on murine calvarial bone. J Periodontol. 2002;73:1141-1148.

6 Stein D, Lee Y, Schmid MJ, Killpack B, Genrich MA, Narayana $\mathrm{N}$, et al. Local simvastatin effects on mandibular bone growth and inflammation. J Periodontol. 2005;76:1861-1870.

7 Rosenblum SF, Frenkel S, Nadkarni P, Ricci JR, Alexander H. Diffusion of fibroblast growth factor from plaster of Paris carrier. J Appl Biomater. 1993;4:67-72.

8 Gombotz WR, Pankey SC, Bouchard LS, Phan DH, Puolakkainen PA. Stimulation of bone healing by transforming growth factor-beta 1 released from polymeric or ceramic implants. J Appl Biometer. 1994;5:141-150.

9 Bai MH, Liu XY, Ge BF, Yallg C, Chen DA. An implant of a composite of bovine bone morphogenetic protein and plaster of Paris for treatment of femoral shaft nonunions. Int Surg. 1996;81:390-392.

10 Dahners LE, Funderburk CH. Gentamycin-loaded plaster of Paris as a treatment of experimental osteomyelitis in rabbits. Clin Orthop. 1987;219:278-282.

11 Mousset B, Benoit MA, Delloye C, Bouillet R, Gillard J. Biodegradable implants for potential use in bone infection: An in vitro study of antibiotic-loaded calcium sulfate. Int Orthop. 1995;19:157-161.

12 Benoit MA, Mousset B, Delloye C, Bouillet R, Gillard J. Antibiotic-loaded plaster of Paris implants coated with poly lactide-co-glycolide as a controlled release delivery system for the treatment of bone infections. Int Orthop. 1997;21:403-408.

13 Turner TM, Urban RM, Gitelis S, Kuo KN, Andersson GB. Radiographic and histologic assessment of calcium sulfate in experimental animal models and clinical use as a resorbable bone-graft substitute, a bone graft expander, and a method for local antibiotic delivery: One institution's experience. J Bone Joint Surg Am. 2001;83:8-18. 